\title{
Relationship between diet quality scores and the risk of frailty and mortality in adults across a wide age spectrum
}

Kulapong Jayanama ${ }^{1,2}$, Olga Theou ${ }^{2,3,4}$, Judith Godin ${ }^{2}$, Leah Cahill ${ }^{4,5}$, Nitin Shivappa ${ }^{6,7,8}$, James R. Hébert ${ }^{6,7,8}$, Michael D. Wirth ${ }^{6,7,8,9}$, Yong-Moon Park ${ }^{10}$, Teresa T. Fung ${ }^{5,11}$ and Kenneth Rockwood ${ }^{2,4^{*}}$ (D)

\begin{abstract}
Background: Beyond intakes of total energy and individual nutrient, eating patterns may influence health, and thereby the risk of adverse outcomes. How different diet measures relate to frailty - a general measure of increased vulnerability to unfavorable health outcomes - and mortality risk, and how this might vary across the life course, is not known. We investigated the associations of five dietary indices (Nutrition Index (NI), the energy-density Dietary Inflammatory Index (E-DII ${ }^{T M}$ ), Healthy Eating Index-2015 (HEI-2015), Mediterranean Diet Score (MDS), and Dietary Approaches to Stop Hypertension (DASH)) with frailty and mortality.
\end{abstract}

Methods: We included 15,249 participants aged $\geq 20$ years from the 2007-2012 cohorts of the National Health and Nutrition Examination Survey (NHANES). The NI combined 31 nutrition-related deficits. The E-DII is a literaturederived dietary index associated with inflammation. The HEl-2015 assesses adherence to the Dietary Guidelines of Americans. The MDS represents adherence to the traditional Mediterranean diet. DASH combines macronutrients and micronutrients to prevent hypertension. Frailty was evaluated using a 36-item frailty index. Mortality status was ascertained up to December 31, 2015.

Results: Participants' mean age was $47.2 \pm 16.7$ years and $51.7 \%$ were women. After adjusting for age, sex, race, educational level, marital and employment status, smoking, BMI, and study cohort, higher NI and E-DII scores and lower HEI-2015, MDS, and DASH scores were individually significantly associated with frailty. All dietary scores were significantly associated with 8-year mortality risk after adjusting for basic covariates and frailty: NI (hazard ratio per 0.1 point, 1.15, 95\%Cl 1.10-1.21), E-DII (per 1 point, 1.05, 1.01-1.08), HEl-2015 (per 10 points, 0.93, 0.89-0.97), MDS (per 1 point, 0.94, 0.90-0.97), and DASH (per 1 point, 0.96, 0.93-0.99). The associations of E-DII, HEl-2015, and MDS scores with 8-year mortality risk persisted after additionally adjusting for $\mathrm{NI}$.

Conclusions: NI, E-DII, HEl-2015, MDS, and DASH scores are associated with frailty and 8-year mortality risk in adults across all ages. Nevertheless, their mechanisms and sensitivity to predict health outcomes may differ. Nutrition scores have the potential to include measures of both consumption and laboratory and physical measures of exposure.

Keywords: Dietary indices, Dietary score, Nutrition, Frailty, Frailty index, Mortality

\footnotetext{
* Correspondence: Kenneth.rockwood@dal.ca

${ }^{2}$ Division of Geriatric Medicine, Dalhousie University \& Nova Scotia Health,

Halifax, Nova Scotia, Canada

${ }^{4}$ Department of Medicine, Dalhousie University, Halifax, Nova Scotia, Canada

Full list of author information is available at the end of the article
}

(c) The Author(s). 2021 Open Access This article is licensed under a Creative Commons Attribution 4.0 International License, which permits use, sharing, adaptation, distribution and reproduction in any medium or format, as long as you give appropriate credit to the original author(s) and the source, provide a link to the Creative Commons licence, and indicate if changes were made. The images or other third party material in this article are included in the article's Creative Commons licence, unless indicated otherwise in a credit line to the material. If material is not included in the article's Creative Commons licence and your intended use is not permitted by statutory regulation or exceeds the permitted use, you will need to obtain permission directly from the copyright holder. To view a copy of this licence, visit http://creativecommons.org/licenses/by/4.0/. The Creative Commons Public Domain Dedication waiver (http://creativecommons.org/publicdomain/zero/1.0/) applies to the data made available in this article, unless otherwise stated in a credit line to the data. 


\section{Background}

Across the lifespan, diet plays an important role in growth, development, and health. Food consumption supplies the energy and essential nutritional substrates required for metabolism and homeostasis. Adequate nutrient intake can decrease the incidence of many diseases and specific deficiencies. However, more subtle imbalances in dietary intake and malnutrition are associated with adverse health outcomes such as metabolic syndrome, type 2 diabetes mellitus, cardiovascular diseases, cancer, infection, cognitive impairment, poor quality of life, disability, and mortality [1-3]. Interestingly, diets that are associated with a lower risk of almost any single disease tend to be associated with lower risk of disease in general, making them "healthy diets" [4].

Despite the convergence of dietary factors associated with lower risk, different diet scores are used to measure different aspects of diet quality. Overall, dietary quality pattern can be more important than individual nutrients in predicting major metabolic and non-communicable diseases [5, 6]. Specifically, the Mediterranean diet, a healthy and balanced dietary pattern focusing heavily on fresh fruits and vegetables, is known to reduce the incidence and mortality rate from major cardiovascular events [4] and cancers [7] and improves cognition in older adults [8]. The Dietary Approaches to Stop Hypertension (DASH), which combines macronutrients and micronutrients, is associated with decreased risk of hypertension and lower cardiovascular-related metabolic diseases [9]. The Healthy Eating Index-2015 (HEI-2015), a diet-quality index, is associated with decreased risk of cancer, cardiovascular disease, and all-cause mortality $[10,11]$. The Dietary Inflammatory Index $\left(\mathrm{DII}^{\circ}\right)$ and energy-adjusted DII (E-DII $\left.{ }^{\mathrm{rm}}\right)$ are literature-derived and population-based scores, which are associated with cancer and a number of other non-communicable chronic diseases [12-17]. More recently, they have also been associated with a higher risk of frailty [18-22].

Frailty, understood as the increased vulnerability to adverse outcomes among people of the same chronological age [23], is related to having higher rates of a variety of diseases and health conditions and is common across the adult-aged spectrum $[24,25]$. Frailty and malnutrition appear to be reciprocally related; higher levels of frailty are associated with malnutrition [26] and malnutrition is related to higher levels of frailty [27]. Given how common frailty is, and how many illnesses themselves are associated with frailty, there is great interest in whether healthy diets can mitigate frailty and its risks. Previously, a multidimensional intervention that included nutrition management was shown to ameliorate frailty [28]. Whether this effect is specific to the intervention and whether particular dietary components drive this effect are unknown. Seeking to understand the relationship between nutrition and frailty better, we developed a Nutrition Index (based on an accumulation of deficits approach) and demonstrated both that it was associated with frailty and that the two together were independently associated with mortality [27]. Similar to other dietary indices, the Nutrition Index consists of nutrients related to health outcomes. In general, poor nutritional status is reflected in some combination of inappropriate intake, disproportionate body composition, and abnormal blood levels. Therefore, the Nutrition Index also includes nutrition-related blood tests and anthropometric measurements.

Although malnutrition is a major marker of frailty, few studies have examined the association of dietary patterns with frailty in older people $[18,29,30]$. Fewer still have evaluated how multiple dietary scores relate to mortality independent of frailty, and across the adult age spectrum. Therefore, the aims of this study are to (1) assess the association between dietary scores and frailty in an adult population unrestricted by age and (2) to explore the impact of these dietary scores on mortality risk after adjusting for the degree of frailty.

\section{Methods}

\section{Study population and design}

This observational study included 17,713 participants aged 20 years or older from the 2007-2008, 2009-2010, and 2011-2012 cohorts of the National Health and Nutrition Examination Survey (NHANES). NHANES is administered by the Centers for Disease Control and Prevention (CDC) and the National Center for Health Statistics (NCHS) and comprises publicly available 2-year cross-sectional surveys that focus on the health and nutrition of noninstitutionalized US residents [31, 32]. Among the 15,287 participants with dietary data, those with missing frailty index scores $(N=15)$ and mortality $(N=23)$ data were excluded from analysis, leaving 15,249 participants with evaluable data.

Each participant provided written informed consent. The NHANES protocol was approved by the institutional review board of the CDC. As a matter of policy, our local Research Ethics Committee does not review secondary analyses of duly approved, publicly available data.

\section{Dietary scores}

Dietary information was assessed using data recalled from the 24 hours $(\mathrm{h})$ prior to the interview. If there were two 24-h recalls available, the first 24-h recall of the providing dietary data was selected for this analysis. Nutrient values derived from the first 24-h recall came from the United States Department of Agriculture (USDA) food composition database. Data from the NHANES dietary interview and the Food Patterns Equivalents Database files were used to calculate the 
dietary scores. The variables used to calculate the dietary scores are presented in Additional file 1: Table S1.

\section{Nutrition Index (NI)}

We previously constructed a deficit accumulation Nutrition Index based from 41 nutrition-related parameters included in the 2003-2006 NHANES cohorts [27, 33]. The Nutrition Index used here equally weighted 31 of these nutrition-related parameters measured in the 20072012 NHANES: 18 nutrients (energy, energy per weight, protein, protein per weight, carbohydrate, percentage of saturated fat, vitamins A, C, B1, B2, B3, and B6, folate, phosphorous, copper, sodium, selenium, fish oil), 3 anthropometric measurements (body mass index, body weight change in the past year, waist circumference), and 10 nutrition-related blood tests (lymphocyte count, hemoglobin, mean corpuscular volume and serum albumin, vitamin $\mathrm{D}$, iron, creatinine, triglyceride, high density lipoprotein (HDL)-cholesterol, and glucose). The Nutrition Index score counts the number of nutritional deficits in an individual in relation to the total deficits considered, therefore yielding values that range between 0 and 1 . A higher score represents worse nutritional status. For a sensitivity analysis, we also split the Nutrition Index into two indices: the Nutrition Index-nutrient, which included only the 18 nutrient items, and the Nutrition Index-lab/exam, which included the 3 anthropometric measurements and the 10 nutrition-related blood tests.

\section{Energy-density Dietary Inflammatory Index (E-DII ${ }^{\mathrm{TM}}$ )}

The E-DII, which is derived using procedures similar to the DII, is a literature-derived dietary index developed by reviewing and scoring 1943 peer reviewed articles that examined the association between 45 dietary parameters and inflammation which were used to derive "inflammatory effect scores". These were then standardized against a global database of intake for the 45 dietary parameters. Full details on the scoring can be found elsewhere [34]. Procedures for computing the E-DII are identical to those used for DII computation except the reference database is itself energy adjusted so that each parameter is expressed per thousand kilocalories (1000 kcal) $[35,36]$. The parameters available for E-DII computation in this study are protein; carbohydrate; total fat; saturated fat; monounsaturated fat; polyunsaturated fat; omega-3 fatty acids; omega- 6 fatty acids; cholesterol; fiber; vitamins A, B1, B2, B3, B6, B12, C, D, and E; folate; beta carotene; iron; magnesium; selenium; zinc; caffeine; and alcohol. A higher score indicates a more proinflammatory dietary intake [37].

\section{Healthy Eating Index-2015 (HEl-2015)}

The HEI-2015 is a diet quality index developed by the USDA's Center for Nutrition Policy and Promotion to assess adherence to the Dietary Guidelines of Americans (DGA) [38]. HEI-2015 has been developed from the HEI-2010 by replacing empty calories with saturated fat and added sugar and focuses on the consumption of total fruits, whole fruits, total vegetables, greens and beans, whole grains, dairy foods, total protein foods, seafood and plant proteins, fatty acids, refined grains, sodium, added sugars, and saturated fats [11]. The score ranges between 0 and 100 points. A higher score reflects healthier eating.

\section{Mediterranean Diet Score (MDS)}

The Mediterranean Diet Score (MDS) assesses adherence to the traditional Mediterranean diet, composed of an abundant consumption of fruits, vegetables, nuts and whole grains, moderate to high amounts of fish and dairy products, low amounts of red meat, consumption of olive oil as the main source of fat, and mild to moderate consumption of wine $[39,40]$. This dietary score includes 10 components: one point score for equal or higher than median intake of non-refined cereals, legumes, fruits and nuts, vegetables (excluding potatoes), fish (shrimp, clams, and fish), and ratio of monounsaturated fatty acids to saturated fatty acids; one point score for lower than median intake of red meat and products, poultry, and dairy products; and one point score for alcohol consumption (14$28 \mathrm{~g} /$ day in female; $28-70 \mathrm{~g} /$ day in male) [41, 42]. Potatoes were excluded in the calculation for NHANES due to differences in preparation methods between the USA and Europe [43]. MDS score ranges between 0 and 10 points and a higher score indicates the better adherence to Mediterranean diet pattern.

\section{Dietary Approaches to Stop Hypertension (DASH)}

The DASH dietary pattern focuses on high amounts of fruits, vegetables, and low-fat dairy products, aimed at lowering blood pressure [44]. Here, we calculated a DASH score based on the nine-item, nutrient-based DASH index: protein, fiber, magnesium, calcium, potassium, total fat, saturated fat, cholesterol, and sodium. Meeting the goal for each component provides one point, meeting an intermediate goal between the DASH diet goal and the nutrient content of the DASH control diet provides 0.5 points, and meeting neither goal gives zero points. The optimal micronutrient targets are energy adjusted per $1000 \mathrm{kcal}$. The control diet targets are from the previous DASH study $[45,46]$. This pattern score ranges between 0 and 9 points, where higher scores indicate greater adherence to the DASH dietary pattern.

\section{Frailty index}

Frailty was evaluated using a 36-item deficit accumulation frailty index modified from previous NHANES studies [25, 27], including self-report health, vital signs, and 
laboratory tests (Additional file 1: Table S2). The frailty index was calculated by counting the number of individual deficits and dividing by the total number of possible deficits. No items related to dietary intake or nutritional status were included in this frailty index. Scores ranged between 0 and 1 , where a higher score is indicative of higher frailty.

\section{Mortality}

Mortality status was identified from the death certificate records in the National Death Index through December 31, 2015 [47]. Survival time was counted from the date of the clinical examination (2007-2012); all participants had between 3 and 8 years of follow-up. We examined mortality rate until 3 years and until 8 years, and time to mortality up to 3 years and up to 8 years.

\section{Statistical analysis}

Participants' characteristics, as a whole and stratified by mortality status, were presented as mean \pm standard deviation (SD) for continuous variables or as frequency (\%) for categorical or ordinal variables. All percentages and means were weighted using the 6-year sampling weights constructed from the sampling weights provided by NHANES for the general US population-based estimates. The correlation between dietary scores was tested using Pearson's correlation $(r)$. If the correlation between dietary scores was not strong, the pairs of dietary scores could be included in the same model. Regarding objective 1 , the association between each dietary score and frailty was analyzed using ordinary least squares (OLS) regression models and we present unstandardized betacoefficients with 95\% confidence intervals (CI) and standardized beta-coefficients. For objective 2, the mortality risk of each dietary score was assessed using Cox regression models, and we present hazard ratios (HR) with the associated 95\% CIs and cumulative survival probability curve. Time to death was tested for both up to 3 years and up to 8 years of follow-up. In both the OLS and Cox regressions, linear and non-linear (squared and cubic) associations were examined. For non-linear associations, the model included the dietary score and the square of the dietary score (allowing a line with one bend), and the cubic model included the dietary score, the square of the dietary score, and the cube of the dietary score (allowing a line with two bends). All regression models were adjusted for potential confounders (provided by NHANES), as basic covariates, including age (continuous, in years), sex (male and female), race (nonHispanic white, non-Hispanic black, Hispanic, and others), education level (less than high school, high school, some college/associated education, and college graduate or higher), marital status (married, widowed, divorced or separated, and never married), employment status (working and non-working), smoking (never, former, and current), body mass index (BMI) $(<18.5 \mathrm{~kg} /$ $\mathrm{m}^{2}, 18.5-24.9,25.0-29.9$, and $\geq 30.0$ ), and study cohort (number). Annual household income was not included as a covariate due to a high rate of missing data (average 9.1\%). Energy intake was included or used for adjusting when creating all dietary scores except MDS. Therefore, energy intake (continuous score in Kcal) was added as a covariate in the regression models where MDS was a single dietary score but not when that model was additionally adjusted for Nutrition Index (Nutrition Index was also adjusted for energy intake). The Cox regression analyses were adjusted for all factors listed above and frailty index (continuous score). To control for the overall nutrition-related accumulation deficits in the association of dietary scores with frailty and mortality we also added the Nutrition Index (continuous score) to our regression models. We also compared all other pairs of dietary scores by running regression models with each combination of two dietary scores to assess which ones more often remained independently associated with the outcome (multicollinearity was tested). The effect of age and sex on the association between each dietary score and frailty and the effect of age, sex, and frailty on the association between each dietary score and mortality were examined using an interaction term, in multivariate OLS and Cox regression analyses, respectively. Statistical significance was considered as $p<0.05$ and all reported probability tests were two-tailed. Statistical analyses were performed using IBM SPSS Statistics for Windows, Version 25.0. Armonk, NY: IBM Corp.

\section{Results}

We analyzed 15,249 participants with complete data, of whom $51.7 \%$ were female. The mean age was $47.2 \pm 16.7$ years and mortality rate up to 8 years was $5.3 \%(N=$ 1171) (Table 1). Older age, female sex, lower energy intake, lower education, non-full-time work, smoking status (former and current), and BMI $<18.5$ or $\geq 25 \mathrm{~kg} / \mathrm{m}^{2}$ were significantly associated with higher frailty (Additional file 1: Table S3). Moderate negative correlations were found between E-DII and HEI-2015 $(r=-0.70)$, and E-DII and DASH $(r=-0.68)$ whereas moderate positive correlations were found between HEI-2015 and MDS $(r=0.60)$, and HEI-2015 and DASH $(r=0.57)$. The correlations between Nutrition Index and the other dietary scores were weak (Fig. 1).

Regarding objective 1 (to assess the association between dietary scores and frailty), higher Nutrition Index and E-DII scores and lower HEI-2015, MDS, and DASH scores were significantly associated with higher frailty (Table 2 and Fig. 2). The HEI-2015, MDS, and DASH were also associated with frailty in the same direction 
Table 1 Descriptive baseline characteristics of participants

\begin{tabular}{|c|c|c|c|}
\hline \multirow{2}{*}{$\begin{array}{l}\text { Characteristics, } \\
\text { mean } \pm \text { SD or } N(\%)\end{array}$} & \multirow{2}{*}{$\begin{array}{l}\text { All } \\
\text { participants }\end{array}$} & \multicolumn{2}{|l|}{ 8-year mortality } \\
\hline & & $\begin{array}{l}\text { Alive } \\
N=14,078\end{array}$ & $\begin{array}{l}\text { Deceased } \\
N=1171\end{array}$ \\
\hline Age (year) & $47.2 \pm 16.7$ & $46.1 \pm 16.1$ & $67.1 \pm 14.7$ \\
\hline Sex, female & $7769(51.7)$ & $7289(52.1)$ & $480(44.9)$ \\
\hline \multicolumn{4}{|l|}{ Race } \\
\hline Non-Hispanic white & $6949(69.2)$ & $6263(68.9)$ & $686(9.3)$ \\
\hline Non-Hispanic black & $3142(10.8)$ & $2907(10.7)$ & $235(75.2)$ \\
\hline Hispanic & $3964(13.6)$ & $3763(13.8)$ & $201(11.3)$ \\
\hline Other & $1194(6.4)$ & $1145(6.5)$ & $49(4.2)$ \\
\hline \multicolumn{4}{|l|}{ Education } \\
\hline Less than high school & $4170(18.2)$ & $3702(17.4)$ & $468(32.4)$ \\
\hline High school & $3480(22.6)$ & $3189(22.4)$ & $291(26.9)$ \\
\hline Some college/ associate education & $4291(30.7)$ & $4031(31.0)$ & $260(25.8)$ \\
\hline College graduate or more & $3291(28.5)$ & $3143(29.2)$ & $148(15.0)$ \\
\hline \multicolumn{4}{|l|}{ Marital status } \\
\hline Married & $9020(63.5)$ & $8444(64.2)$ & $576(50.7)$ \\
\hline Widowed & $1276(5.7)$ & $946(4.6)$ & $330(26.5)$ \\
\hline Divorced or separated & 2179 (12.6) & $2002(12.5)$ & $177(14.5)$ \\
\hline Never married & $2769(18.1)$ & $2681(18.7)$ & $88(8.3)$ \\
\hline Full-time working & $8278(63.0)$ & 8069 (65.3) & $209(22.7)$ \\
\hline \multicolumn{4}{|l|}{ Smoking status } \\
\hline Never & $8253(54.6)$ & $7771(55.3)$ & $482(42.1)$ \\
\hline Former & $3739(24.7)$ & $3268(23.9)$ & $471(38.7)$ \\
\hline Current & $3252(20.7)$ & $3034(20.8)$ & $218(19.2)$ \\
\hline \multicolumn{4}{|l|}{ Body mass index $\left(\mathrm{kg} / \mathrm{m}^{2}\right)$} \\
\hline$<18.5$ & $251(1.7)$ & $221(1.6)$ & $30(2.9)$ \\
\hline $18.5-24.9$ & $4174(29.5)$ & 3864 (29.6) & $310(27.8)$ \\
\hline $25.0-29.9$ & $5071(33.9)$ & 4668 (33.8) & $403(34.7)$ \\
\hline$\geq 30$ & $5595(34.9)$ & $5224(35.0)$ & $371(34.6)$ \\
\hline Energy intake (Kcal) & $2182.3 \pm 999.1$ & $2199.1 \pm 1000.6$ & $1882.7 \pm 920.8$ \\
\hline $\mathrm{Nl}$ score (0 to 1$)$ & $0.34 \pm 0.15$ & $0.33 \pm 0.15$ & $0.41 \pm 0.16$ \\
\hline E-DII score $(-5.81$ to 4.82$)$ & $0.35 \pm 1.97$ & $0.37 \pm 1.97$ & $0.15 \pm 1.92$ \\
\hline HEl-2015 score (0 to 100$)$ & $51.2 \pm 13.6$ & $51.1 \pm 13.6$ & $51.8 \pm 13.1$ \\
\hline MDS (0 to 10$)$ & $4.0 \pm 1.6$ & $4.0 \pm 1.6$ & $4.0 \pm 1.5$ \\
\hline DASH score (0 to 9) & $2.5 \pm 1.6$ & $2.5 \pm 1.5$ & $2.5 \pm 1.6$ \\
\hline FI score (0 to 1$)$ & $0.10 \pm 0.10$ & $0.09 \pm 0.09$ & $0.24 \pm 0.15$ \\
\hline
\end{tabular}

DASH Dietary Approaches to Stop Hypertension, E-DII Energy-density Dietary Inflammatory Index, FI Frailty index, HEl-2015 Healthy Eating Index-2015, Kcal kilocalories, $k g$ kilogram, $m$ meter, MDS Mediterranean Diet Score, NI Nutrition Index. Higher NI and E-DII scores and lower HEl-2015, MDS, and DASH scores represent worse dietary pattern/intake. The percentages and mean values are weighted with sampling weights

after controlling for the Nutrition Index; Nutrition Index remained a significant predictor.

Regarding objective 2 (to assess the impact of these dietary scores on mortality risk after adjusting for the degree of frailty), higher Nutrition Index, lower HEI2015, and lower MDS scores were associated with 3-year mortality and all dietary scores (higher Nutrition Index and E-DII, and lower HEI-2015, MDS, and DASH scores) were associated with 8-year mortality risk; these results persisted after adjusting for the frailty index. After controlling for the Nutrition Index, none of the scores were associated with 3-year mortality, however the E-DII, HEI-2015 and MDS scores were significantly associated with 8-year mortality. The Nutrition Index 

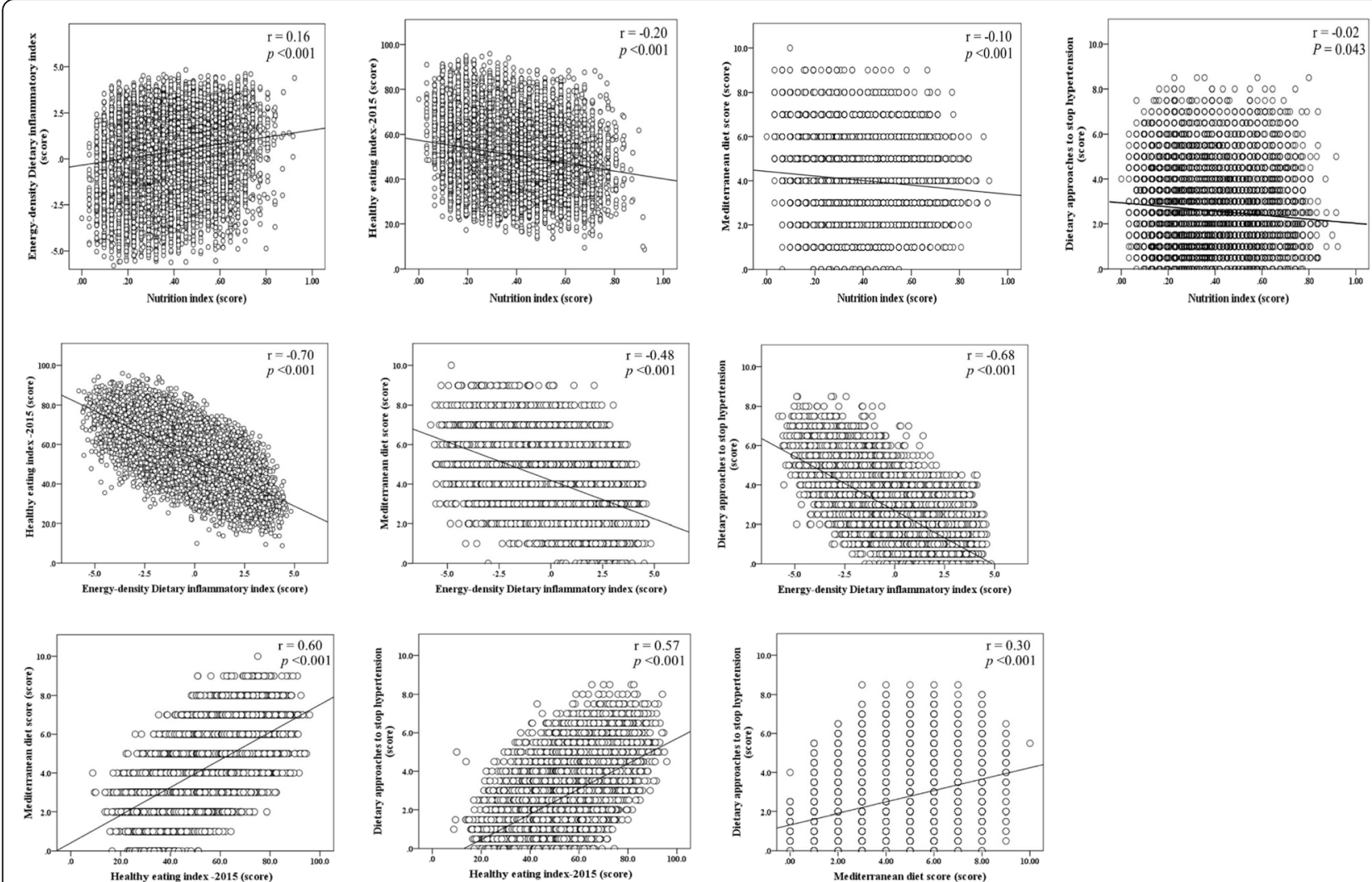

Fig. 1 Bivariate scatter plots and linear regression lines between each pair of the dietary scores. Higher NI and E-DII scores and lower HEl-2015, MDS, and DASH scores represent worse dietary pattern/intake

remained a significant predictor of 3- and 8-year mortality in all Cox regression models. When we compared the other pair of dietary scores, none of them predicted 3year mortality independently. HEI-2015 and MDS remained significant predictors of 8-year mortality except for the model where they were both added together, and E-DII remained a significant predictor of 8-year mortality except when it was included in the same model with DASH (Table 3).

For a sensitivity analysis, we examined each component of Nutrition Index (Nutrition Index-nutrient and Nutrition Index-lab/exam) and found that both of these indices were associated with higher level of frailty after adjusting for basic covariates. Correlations between $\mathrm{Nu}$ trition Index-nutrient and Nutrition Index-lab/exam and the other dietary scores were weak (Additional file 1: Table S4). All dietary scores were significantly associated with frailty after controlling for both Nutrition Indexnutrient and Nutrition Index-lab/exam, except E-DII (Additional file 1: Table S5). Both Nutrition indices (nutrient and lab/exam) were associated with 8-year mortality risk and the Nutrition Index-lab/exam was further associated with 3-year mortality risk. An association with 8-year mortality risk was found with the E-DII, HEI2015, and MDS after controlling for Nutrition Index- nutrient or Nutrition Index-lab/exam but only in MDS after controlling for both Nutrition Index-nutrient and Nutrition Index-lab/exam (Additional file 1: Table S6).

In both the OLS and Cox regression models, there were no interactions of age and sex with the association between each dietary score and frailty (Additional file 1: Table S7), nor any interactions of age, sex, and frailty with the association between each dietary score and mortality (Additional file 1: Table S8).

\section{Discussion}

This observational study examined the relationship of five dietary scores (Nutrition Index, E-DII, HEI-2015, MDS, and DASH) with frailty measured using a frailty index and with mortality after having considered the degree of health deficit accumulation in adults of all ages. We found that each dietary measure (higher Nutrition Index and E-DII scores, and lower HEI-2015, MDS, and DASH scores) was associated with higher frailty risk. When controlling for frailty, adherence to dietary scores was associated with 3-year mortality risk (short-term), but here the result depended more on the measure, being seen only with higher Nutrition Index score, and lower HEI-2015 and MDS scores. The association between dietary scores and greater risk of death was more 
Table 2 Relationship between dietary scores and frailty, using multivariable-adjusted ordinary least squares regression analyses

\begin{tabular}{|c|c|c|c|}
\hline Dietary scores & Unstandardized beta-coefficients $(95 \% \mathrm{Cl})$ & Standardized beta-coefficients & $p$ value \\
\hline $\mathrm{NI}$ (per 0.1 point) & 0.014 (0.002 to 0.025$)$ & 0.19 & 0.017 \\
\hline NI squared & $-0.003(-0.006$ to 0.000$)$ & -0.32 & 0.061 \\
\hline $\mathrm{Nl}$ cubic & 0.000 (0.000 to 0.001$)$ & 0.26 & 0.008 \\
\hline E-DII (per 1 point) & 0.002 (0.001 to 0.002$)$ & 0.03 & $<0.001$ \\
\hline HEI-2015 (per 10 points) & $0.003(-0.003$ to 0.010$)$ & 0.04 & 0.307 \\
\hline HEI-2015 squared & $-0.001(-0.001$ to 0.000$)$ & -0.09 & 0.022 \\
\hline MDS (per 1 point)* & $-0.003(-0.004$ to -0.002$)$ & -0.05 & $<0.001$ \\
\hline DASH (per 1 point) & $-0.001(-0.002$ to -0.001$)$ & -0.02 & 0.003 \\
\hline E-DII (per 1 point) & 0.000 (0.000 to 0.001$)$ & 0.01 & 0.293 \\
\hline $\mathrm{NI}$ (per 0.1 point) & 0.008 (0.007 to 0.009$)$ & 0.12 & $<0.001$ \\
\hline HEl-2015 (per 10 points) & 0.006 (0.000 to 0.012$)$ & 0.08 & 0.048 \\
\hline HEI-2015 squared & $-0.001(-0.001$ to 0.000$)$ & -0.10 & 0.008 \\
\hline $\mathrm{NI}$ (per 0.1 point) & 0.008 (0.007 to 0.009 ) & 0.11 & $<0.001$ \\
\hline MDS (per 1 point) & $-0.002(-0.003$ to -0.001$)$ & -0.03 & $<0.001$ \\
\hline $\mathrm{NI}$ (per 0.1 point) & 0.011 (0.010 to 0.012 ) & 0.11 & $<0.001$ \\
\hline DASH (per 1 point) & $-0.001(-0.002$ to -0.001$)$ & -0.02 & 0.002 \\
\hline $\mathrm{NI}$ (per 0.1 point) & 0.018 (0.007 to 0.019$)$ & 0.12 & $<0.001$ \\
\hline HEl-2015 (per 10 points) & $-0.004(-0.006$ to -0.003$)$ & -0.05 & $<0.001$ \\
\hline E-DII (per 1 point) & 0.000 (0.000 to 0.001$)$ & -0.01 & 0.427 \\
\hline MDS (per 1 point) & $-0.003(-0.004$ to -0.002$)$ & -0.04 & $<0.001$ \\
\hline E-DII (per 1 point) & 0.000 (0.000 to 0.001$)$ & 0.01 & 0.190 \\
\hline DASH (per 1 point) & $0.000(-0.001$ to 0.001$)$ & 0.00 & 0.916 \\
\hline E-DII (per 1 point) & 0.002 (0.001 to 0.003 ) & 0.03 & 0.002 \\
\hline MDS (per 1 point) & $-0.002(-0.003$ to 0.000$)$ & -0.03 & 0.001 \\
\hline HEI-2015 (per 10 points) & $-0.003(-0.004$ to -0.001$)$ & -0.03 & $<0.001$ \\
\hline DASH (per 1 point) & 0.001 (0.000 to 0.002) & 0.01 & 0.193 \\
\hline HEI-2015 (per 10 points) & $-0.004(-0.006$ to -0.003$)$ & -0.05 & $<0.001$ \\
\hline DASH (per 1 point) & $0.000(-0.001$ to 0.000$)$ & -0.01 & 0.302 \\
\hline MDS (per 1 point) & $-0.003(-0.004$ to -0.002$)$ & -0.04 & $<0.001$ \\
\hline
\end{tabular}

Higher NI and E-DII scores and lower HEl-2015, MDS, and DASH scores represent worse dietary pattern/intake. All regression models were adjusted for age, sex, race, educational level, marital status, employment status, smoking, study cohort and BMI. In an initial model we tested the linear relationship, in the second model we added the squared term, and in the third model we added the cubic term. We present results only for the highest order model that was statistically significant. If none of the models were statistically significant, we present the linear model

$B M I$ body mass index, DASH Dietary Approaches to Stop Hypertension, E-DII Energy-density Dietary Inflammatory Index, FI Frailty index, HEl-2015 Healthy Eating Index-2015, MDS Mediterranean Diet Score, NI Nutrition Index

*This regression model was additionally adjusted for energy intake. The standardized beta-coefficients were calculated by multiplying the unstandardized coefficient by the ratio of the standard deviations of the dietary scores and frailty index 

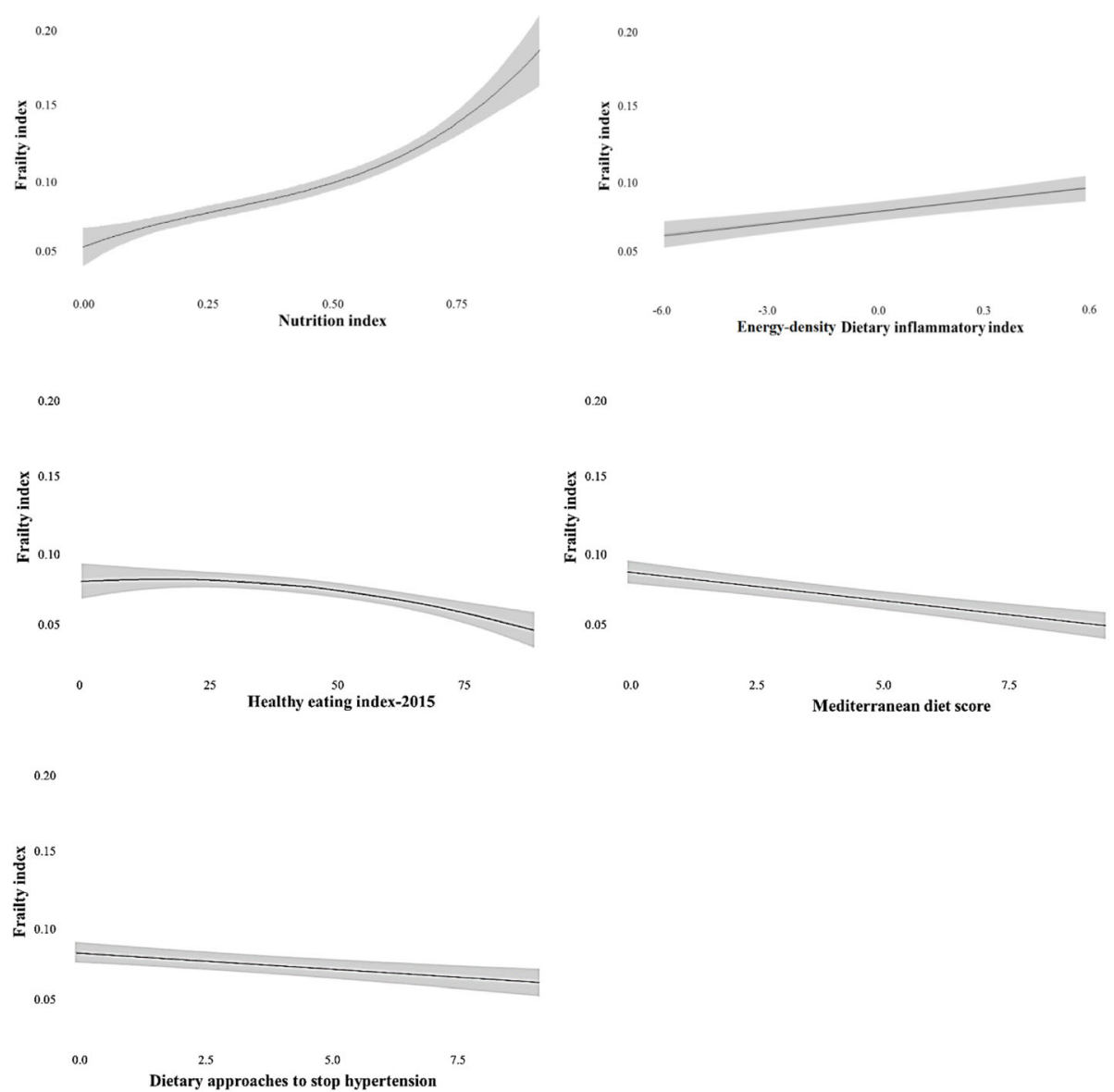

Fig. 2 Relationship between dietary scores and frailty. Here we present frailty index predictive values with 95\% confidence interval for each level of dietary scores. Regression models were adjusted for basic covariates (age, sex, race, education level, marital status, employment status, smoking, study cohort, and body mass index). Higher Nutrition Index and Energy-density Dietary Inflammatory Index scores and lower Healthy Eating Index-2015, Mediterranean Diet Score, and Dietary Approaches to Stop Hypertension scores represent worse dietary pattern/intake

robust for 8-year (medium-term) mortality, elicited among all measures of nutrition intake, i.e., higher Nutrition Index and E-DII scores, and lower HEI-2015, MDS, and DASH scores. (Table 4).

Similar to our previous study [27], we found that higher (worse) Nutrition Index score-which measures both intake and nutritional status-was significantly associated with higher frailty and with higher mortality risk independent of frailty. Even controlling for the degree of frailty, the Nutrition Index predicted both shortterm and medium-term mortality risk. As health deficits accumulate, poor nutritional status could be a factor of frailty. For this reason, we excluded items related to dietary intake or nutritional status from the frailty index. While the Nutrition Index included serum glucose, triglyceride, and HDL-cholesterol, it did not assess underlying diseases related to metabolic syndrome (e.g., diabetes mellitus, high blood pressure) that were included in the frailty index. Patients diagnosed with diabetes mellitus, appropriate dietary intake, and well- controlled serum glucose could have better nutritional status and lower adverse outcomes comparing those with poor-controlled diet intake and serum glucose. This emphasizes that optimized nutritional interventions could modify the Nutrition Index and therefore influence clinical outcomes.

Of the two components of the Nutrition Index, the Nutrition Index-lab/exam was more strongly associated with mortality than the Nutrition Index-nutrient component, which reflected consumption. Moreover, the Nutrition Index-lab/exam was the strongest predictor of 3-year and 8-year mortality risk. The quantity of dietary intake is important and relates to worsening health outcomes. Inasmuch as blood tests and physical examinations (especially anthropometric measurements) yield estimates with narrower variances, they may allow earlier identification of health deficit accumulations and better detection of abnormal physiological status beyond malnutrition. Therefore, blood tests and physical examinations may be more closely related to frailty and can be 
Table 3 Relationship between dietary scores and mortality, using multivariable-adjusted Cox regression analysis

\begin{tabular}{|c|c|c|c|c|}
\hline \multirow[t]{2}{*}{ Dietary scores } & \multicolumn{2}{|c|}{$\begin{array}{l}\text { 3-year mortality } \\
\text { Deceased, } N(\%)=509(2.4)\end{array}$} & \multicolumn{2}{|c|}{$\begin{array}{l}\text { 8-year mortality } \\
\text { Deceased, } N(\%)=1171(5.3)\end{array}$} \\
\hline & Hazard ratio $(95 \% \mathrm{Cl})$ & $p$ value & Hazard ratio $(95 \% \mathrm{Cl})$ & $p$ value \\
\hline \multicolumn{5}{|c|}{ Adjusted for age, sex, race, educational level, marital status, employment status, smoking, study cohort, and BMI (basic covariates) } \\
\hline $\mathrm{NI}$ (per 0.1 point) & $1.16(1.09,1.22)$ & $<0.001$ & $1.14(1.09,1.18)$ & $<0.001$ \\
\hline E-DII (per 1 point) & $1.03(0.98,1.08)$ & 0.201 & $1.04(1.01,1.08)$ & 0.008 \\
\hline HEI-2015 (per 10 points) & $0.92(0.86,0.98)$ & 0.015 & $0.92(0.88,0.96)$ & $<0.001$ \\
\hline MDS (per 1 point)* & $0.93(0.87,0.98)$ & 0.013 & $0.93(0.89,0.96)$ & $<0.001$ \\
\hline DASH (per 1 point) & $0.96(0.90,1.01)$ & 0.121 & $0.96(0.93,0.99)$ & 0.038 \\
\hline \multicolumn{5}{|c|}{ Adjusted for basic covariates and FI } \\
\hline $\mathrm{NI}$ (per 0.1 point) & $1.11(1.04,1.17)$ & 0.001 & $1.15(1.10,1.21)$ & $<0.001$ \\
\hline E-DII (per 1 point) & $1.03(0.99,1.08)$ & 0.185 & $1.05(1.01,1.08)$ & 0.007 \\
\hline HEl-2015 (per 10 points) & $0.93(0.86,0.99)$ & 0.028 & $0.93(0.89,0.97)$ & 0.002 \\
\hline MDS (per 1 point)* & $0.94(0.88,0.99)$ & 0.046 & $0.94(0.90,0.97)$ & 0.001 \\
\hline DASH (per 1 point) & $0.96(0.90,1.01)$ & 0.113 & $0.96(0.93,0.99)$ & 0.040 \\
\hline E-DII (per 1 point) & $1.01(0.96,1.06)$ & 0.791 & $1.02(0.99,1.06)$ & 0.198 \\
\hline E-DII squared & $0.98(0.96,1.00)$ & 0.072 & $0.99(0.97,0.99)$ & 0.043 \\
\hline $\mathrm{NI}$ (per 0.1 point) & $1.10(1.04,1.17)$ & 0.002 & $1.08(1.04,1.13)$ & $<0.001$ \\
\hline HEI-2015 (per 10 points) & $0.95(0.88,1.02)$ & 0.164 & $0.95(0.91,0.99)$ & 0.037 \\
\hline $\mathrm{NI}$ (per 0.1 point) & $1.09(1.04,1.16)$ & 0.005 & $1.08(1.04,1.12)$ & $<0.001$ \\
\hline MDS (per 1 point) & $0.95(0.89,1.01)$ & 0.106 & $0.94(0.91,0.98)$ & 0.004 \\
\hline $\mathrm{NI}$ (per 0.1 point) & $1.10(1.04,1.17)$ & 0.002 & $1.08(1.04,1.13)$ & $<0.001$ \\
\hline DASH (per 1 point) & $0.96(0.91,1.02)$ & 0.166 & $0.97(0.93,1.00)$ & 0.062 \\
\hline $\mathrm{NI}$ (per 0.1 point) & $1.10(1.04,1.17)$ & 0.001 & $1.09(1.05,1.13)$ & $<0.001$ \\
\hline HEI-2015 (per 10 points) & $0.91(0.83,1.00)$ & 0.057 & $0.94(0.88,0.99)$ & 0.045 \\
\hline E-DII (per 1 point) & $0.98(0.92,1.05)$ & 0.595 & $1.01(0.97,1.05)$ & 0.724 \\
\hline E-DII squared & $0.98(0.96,1.00)$ & 0.057 & $0.99(0.97,0.99)$ & 0.036 \\
\hline MDS (per 1 point) & $0.94(0.88,1.01)$ & 0.083 & $0.94(0.90,0.99)$ & 0.009 \\
\hline E-DII (per 1 point) & $1.01(0.95,1.06)$ & 0.855 & $1.02(0.98,1.06)$ & 0.309 \\
\hline E-DII squared & $0.98(0.96,1.00)$ & 0.058 & $0.99(0.97,0.99)$ & 0.033 \\
\hline DASH (per 1 point) & $0.97(0.90,1.05)$ & 0.411 & $0.99(0.94,1.04)$ & 0.735 \\
\hline E-DII (per 1 point) & $1.01(0.95,1.08)$ & 0.689 & $1.04(0.99,1.08)$ & 0.090 \\
\hline MDS (per 1 point) & $0.97(0.90,1.04)$ & 0.352 & $0.96(0.91,1.00)$ & 0.059 \\
\hline HEI-2015 (per 10 points) & $0.95(0.87,1.03)$ & 0.188 & $0.96(0.91,1.01)$ & 0.113 \\
\hline DASH (per 1 point) & $0.99(0.92,1.06)$ & 0.741 & $0.99(0.95,1.04)$ & 0.728 \\
\hline HEl-2015 (per 10 points) & $0.93(0.86,1.01)$ & 0.102 & $0.94(0.87,0.99)$ & 0.016 \\
\hline
\end{tabular}


Table 3 Relationship between dietary scores and mortality, using multivariable-adjusted Cox regression analysis (Continued)

\begin{tabular}{|c|c|c|c|c|}
\hline \multirow[t]{2}{*}{ Dietary scores } & \multicolumn{2}{|c|}{$\begin{array}{l}\text { 3-year mortality } \\
\text { Deceased, } N(\%)=509(2.4)\end{array}$} & \multicolumn{2}{|c|}{$\begin{array}{l}\text { 8-year mortality } \\
\text { Deceased, } N(\%)=1171(5.3)\end{array}$} \\
\hline & Hazard ratio $(95 \% \mathrm{Cl})$ & $p$ value & Hazard ratio $(95 \% \mathrm{Cl})$ & $p$ value \\
\hline DASH (per 1 point) & $0.97(0.91,1.03)$ & 0.290 & $0.98(0.94,1.01)$ & 0.193 \\
\hline MDS (per 1 point) & $0.95(0.98,1.01)$ & 0.093 & $0.94(0.90,0.98)$ & 0.004 \\
\hline
\end{tabular}

2007-2012 NHANES cohorts were included in the analysis and mortality was identified up to December 2015. Higher NI and E-DII scores and lower HEl-2015, MDS, and DASH scores represents worse dietary pattern/intake. In an initial model we tested the linear relationship, in the second model we added the squared term, and in the third model, we added the cubic term. We present results only for the highest order model that was statistically significant. If none of the models were statistically significant, we present the linear model

BMI body mass index, DASH Dietary Approaches to Stop Hypertension, E-DII Energy-density Dietary Inflammatory Index, Fl Frailty index, HEl-2015 Healthy Eating Index-2015, MDS Mediterranean Diet Score, NI Nutrition Index

*This regression model was additionally adjusted for energy intake

a more sensitive predictor of mortality [37, 48]. However, nutrient consumption was based on a single 24-h recall and people could inaccurately remember the amount of a food that they ate. For these reasons, all nutrition-related parameters including nutrients, blood tests, and physical exams should be considered when investigating the relationship between nutrition and health outcomes.

The present study revealed associations of higher EDII and lower HEI-2015, MDS, and DASH scores with higher frailty risk (using frailty index) and higher 8-year mortality risk when controlling for frailty. Lower HEI2015 and MDS scores were associated with higher 3year mortality risk. In addition, we found associations of HEI-2015, MDS, and DASH scores with frailty after controlling for the Nutrition Index. We also found associations of HEI-2015, and MDS with medium-term (8-year) mortality after controlling for the Nutrition Index. These findings support earlier studies [11, 20, 22, 40, 49-53] that reported relationships between these scores and adverse health outcomes including cardiovascular disease, brain health, cancer, frailty phenotype, and mortality. These varied dietary scores have similar relationships with frailty and mortality due to the inclusion of healthy nutrients. Notably, the pathophysiological links of these dietary scores to frailty and mortality are different; higher E-DII is associated with higher inflammatory status [54, 55]; higher HEI-2015 score, representing overall diet quality following USDA and DGA recommendations, and Mediterranean-style diet (MDS) are related to sarcopenia and age-related chronic disease "prevention" [56]; and higher DASH score improves blood pressure, lipid profile, and inflammatory status [57]. The independent association of HEI-2015, MDS consumption, and the Nutrition Index with frailty and mortality may reflect that these dietary scores are individually important and their pathogenesis in frailty and mortality could differ. For example, the association of Nutrition Index score could be related to the current nutritional status (adequate intake and nourishment) and the association of HEI-2015 and MDS could result from the balance and nutrient-specific effect of a healthy diet.

Table 4 Summary of the associations between each dietary score and frailty and between each dietary score and mortality

\begin{tabular}{|c|c|c|c|c|c|c|}
\hline \multirow{2}{*}{$\begin{array}{l}\text { Dietary } \\
\text { scores }\end{array}$} & \multicolumn{2}{|l|}{ Frailty } & \multicolumn{2}{|l|}{ 3-year mortality } & \multicolumn{2}{|l|}{ 8-year mortality } \\
\hline & $\begin{array}{l}\text { Adjusted for } \\
\text { covariates }\end{array}$ & $\begin{array}{l}\text { Adjusted for } \\
\text { covariates + NI }\end{array}$ & $\begin{array}{l}\text { Adjusted for } \\
\text { covariates + FI }\end{array}$ & $\begin{array}{l}\text { Adjusted for } \\
\text { covariates + FI + NI }\end{array}$ & $\begin{array}{l}\text { Adjusted for } \\
\text { covariates + FI }\end{array}$ & $\begin{array}{l}\text { Adjusted for } \\
\text { covariates }+\mathrm{FI}+\mathrm{NI}\end{array}$ \\
\hline $\mathrm{NI}$ & Non-linear & N/A & + & N/A & + & N/A \\
\hline E-DII & + & $x$ & $x$ & $x$ & + & Non-linear \\
\hline HEl-2015 & Non-linear & Non-linear & - & $x$ & - & - \\
\hline MDS & - & - & - & $x$ & - & - \\
\hline DASH & - & - & $x$ & $x$ & - & $x$ \\
\hline $\begin{array}{l}\mathrm{Nl}- \\
\text { nutrient }\end{array}$ & + & N/A & $x$ & N/A & + & N/A \\
\hline $\begin{array}{l}\mathrm{NI}-\mathrm{lab} / \\
\text { exam }\end{array}$ & Non-linear & N/A & + & N/A & + & N/A \\
\hline
\end{tabular}

+ positive association, - reverse association, $\mathrm{X}$ no association, N/A not applicable

2007-2012 NHANES cohorts were included in the analysis and mortality was identified up to December 2015. We separated the 31-item nutrition Index into two indices: the NI-nutrient which included only the 18 nutrients and the Nl-lab/exam which included 10 nutrition-related blood tests and 3 anthropometric measurements. Higher NI and E-DII scores and lower HEI-2015, MDS, and DASH scores represents worse dietary pattern/intake. Covariates in regression models and Cox regression models were age, sex, race, educational level, marital status, employment status, smoking, study cohort and BMI. The actual scores can be found in Tables 2 and 3, and Additional file 1: Table S5 and S6

DASH Dietary Approaches to Stop Hypertension, E-DIl Energy-density Dietary Inflammatory Index, FI Frailty index, HEl-2015 Healthy Eating Index-2015, MDS Mediterranean Diet Score, NI Nutrition Index 
This study examined the publicly available and large population-based NHANES data in which the protocol was rigorous and well-controlled. Dietary data were systematically recorded and calculated. Mortality was extracted from death certificate data and had a long follow-up period. All analyses were controlled for various potential confounding factors. Nonetheless, there are some limitations to be aware of when interpreting these results: (1) NHANES is a cross-sectional design and the causal relationship between frailty and nutrition cannot be evaluated directly because of the inability to evaluate temporal relationships in the data; (2) dietary data were recorded using 24-h recall, so day-to-day variation could not be measured, and food intake could have changed during the study period and therefore recall data may not represent participants' long-term dietary patterns; (3) the estimates of dietary scores were based on self-reported dietary data, not including supplement use; and (4) reverse causation could be possible, as dietary consumption and nutritional status may be altered when people start to feel unwell. Future randomized controlled trials are required to confirm the effect of the change in each dietary score on frailty and mortality risk.

\section{Conclusions}

Even though adequate energy and protein intake are essential, and some macronutrients and micronutrients are related to frailty and mortality, overall daily dietary intakes-including more than one nutrient as an eating pattern-should be taken into consideration. This study revealed that the Nutrition Index, E-DII, HEI-2015, MDS, and DASH were associated with frailty and mortality risk across the life course. Nevertheless, their mechanisms within health outcomes may differ. Regarding the Nutrition Index, the accumulation of blood test and physical examination deficits may be a more sensitive predictor of adverse health outcomes such as frailty and mortality, compared to the accumulation of deficits solely regarding dietary intake.

\section{Supplementary Information}

The online version contains supplementary material available at https://doi. org/10.1186/s12916-021-01918-5.

Additional file 1: Table S1. Dietary variables included in calculations of the dietary scores. Table S2. 36-item frailty index. Table S3. Association of participants' baseline characteristics with frailty, using univariate linear regression analyses and with mortality risk, using Cox regression analysis. Table S4. Correlation of the Nutrition Index-nutrient and Nutrition Indexlab/exam with other dietary scores. Table S5. Relationship of the Nutrition Index-nutrient and Nutrition Index-lab/exam with frailty, using multivariable-adjusted ordinary least squares regression analyses. Table S6. Relationship of the Nutrition Index-nutrient and Nutrition Index-lab/ exam with mortality, using multivariable-adjusted Cox regression analysis. Table S7. Coefficients and $p$ values for the 3-way interactions of each dietary score with age and sex on frailty. Table S8. Coefficients and $p$ values for the 4-way interactions of each dietary score with age, sex, and frailty on mortality.

\section{Abbreviations}

BMl: Body mass index; CDC: Centers for Disease Control and Prevention; Cl: Confidence interval; DASH: Dietary Approaches to Stop Hypertension; DGA: Dietary Guidelines of Americans; E-DIl: Energy-density Dietary Inflammatory Index; HEl-2015: Healthy Eating Index-2015; HR: Hazard ratio; kg: Kilogram; m: Meter; MDS: Mediterranean Diet Score; NCHS: National Center for Health Statistics; NHANES: National Health and Nutrition Examination Survey; OLS: Ordinary least squares; USDA: United States Department of Agriculture

\section{Acknowledgements}

We are grateful to the Faculty of Medicine Ramathibodi Hospital, Mahidol University, for supporting $\mathrm{KJ}$ with a research fellowship to conduct this research; our colleagues at Geriatric Medicine Research, Dalhousie University/ Nova Scotia Health for their support; all NHANES participants; and the NHANES researchers for making this data publicly available.

\section{Authors' contributions}

$\mathrm{KJ}, \mathrm{OT}$, and KR conceived and designed the study, interpreted the data, and drafted the manuscript. JG assisted with data analysis, interpreted the data, and revised the manuscript. LC designed the study and revised the manuscript. NS, JRH, MDW, YP, and TTF assisted with data analysis and revised the manuscript. All authors critically revised the manuscript, agree to be fully accountable for ensuring the integrity and accuracy of the work, and read and approved the final manuscript before submission.

Funding

This study was not funded entirely or partially by an outside source.

Availability of data and materials

The National Health and Nutrition Examination Survey (NHANES) data are publicly available at https://www.cdc.gov/nchs/nhanes/index.htm.

\section{Ethics approval and consent to participate}

The protocols of NHANES were approved by the institutional review board of the National Center for Health Statistics, CDC. Written informed consent was obtained from each participant before participation in this study.

Consent for publication

Not applicable.

\section{Competing interests}

Dr. James R. Hébert owns controlling interest in Connecting Health Innovations LLC (CHI), a company that has licensed the right to his invention of the dietary inflammatory index $\left(\mathrm{D} \|^{\circledR}\right)$ from the University of South Carolina in order to develop computer and smart phone applications for patient counseling and dietary intervention in clinical settings. Drs. Nitin Shivappa and Michael Wirth are employees of $\mathrm{CHI}$. The subject matter of this paper will not have any direct bearing on that work, nor has that activity exerted any influence on this project.

Dr. Kenneth Rockwood is Chief Science Officer of DGl Clinical, which in the last 5 years has contracts with pharma and device manufacturers (Baxter, Baxalta, Shire, Hollister, Nutricia, Roche, Otsuka) on individualized outcome measurement. In 2017, he attended an advisory board meeting with Lundbeck and in 2019 another with Nutricia. Otherwise, any personal fees are for invited guest lectures and academic symposia, received directly from event organizers, chiefly for presentations on frailty. He is Associate Director of the Canadian Consortium on Neurodegeneration in Aging, which is funded by the Canadian Institutes of Health Research, and with additional funding from the Alzheimer Society of Canada and several other charities. Other authors declare no potential competing interests.

\section{Author details}

${ }^{1}$ Chakri Naruebodindra Medical Institute, Faculty of Medicine Ramathibodi Hospital, Mahidol University, Samut Prakan, Thailand. ${ }^{2}$ Division of Geriatric Medicine, Dalhousie University \& Nova Scotia Health, Halifax, Nova Scotia, 
Canada. ${ }^{3}$ School of Physiotherapy, Dalhousie University, Halifax, Nova Scotia, Canada. ${ }^{4}$ Department of Medicine, Dalhousie University, Halifax, Nova Scotia, Canada. ${ }^{5}$ Department of Nutrition, Harvard T.H. Chan School of Public Health, Boston, MA, USA. ${ }^{6}$ Cancer Prevention and Control Program, University of South Carolina, Columbia, SC, USA. ${ }^{7}$ Department of Epidemiology and Biostatistics, Arnold School of Public Health, University of South Carolina, Columbia, SC, USA. ${ }^{8}$ Department of Nutrition, Connecting Health Innovations LLC, Columbia, SC, USA. ' ${ }^{9}$ College of Nursing, University of South Carolina, Columbia, SC, USA. ${ }^{10}$ Department of Epidemiology, Fay W. Boozman College of Public Health, University of Arkansas for Medical Sciences, Little Rock, AR, USA. ${ }^{11}$ Department of Nutrition, Simmons University, Boston, MA, USA.

Received: 29 September 2020 Accepted: 19 January 2021 Published online: 16 March 2021

\section{References}

1. Ribeiro RV, Hirani V, Senior AM, Gosby AK, Cumming RG, Blyth FM, et al. Diet quality and its implications on the cardio-metabolic, physical and general health of older men: the Concord Health and Ageing in Men Project (CHAMP). Br J Nutr. 2017;118(2):130-43. https://doi.org/10.1017/S0007114517001738.

2. Ruengurairoek $T$, Vathesatogkit $P$, Boonhat $H$, Warodomwichit $D$, Thongmuang N, Matchariyakul $\mathrm{D}$, et al. The association between processed meat intake and the prevalence of type 2 diabetes in Thais: a crosssectional study from the Electricity Generating Authority of Thailand. Rama Med J. 2017:40:1-10.

3. Rasheed S, Woods RT. Malnutrition and quality of life in older people: a systematic review and meta-analysis. Ageing Res Rev. 2013;12(2):561-6. https://doi.org/10.1016/j.arr.2012.11.003.

4. Estruch R, Ros E, Salas-Salvado J, Covas Ml, Corella D, Aros F, et al. Primary prevention of cardiovascular disease with a Mediterranean diet supplemented with extra-virgin olive oil or nuts. N Engl J Med. 2018;378(25):e34.

5. Reedy J, Krebs-Smith SM, Miller PE, Liese AD, Kahle LL, Park Y, et al. Higher diet quality is associated with decreased risk of all-cause, cardiovascular disease, and cancer mortality among older adults. J Nutr. 2014;144(6):881-9.

6. Cespedes EM, Hu FB, Tinker L, Rosner B, Redline S, Garcia L, et al. Multiple healthful dietary patterns and type 2 diabetes in the women's health initiative. Am J Epidemiol. 2016:183(7):622-33.

7. Fresan U, Sabate J, Martinez-Gonzalez MA, Segovia-Siapco G, de la FuenteArrillaga C, Bes-Rastrollo M. Adherence to the 2015 dietary guidelines for Americans and mortality risk in a Mediterranean cohort: the SUN project. Prev Med. 2019;1 18:317-24. https://doi.org/10.1016/j.ypmed.2018.11.015.

8. Valls-Pedret C, Sala-Vila A, Serra-Mir M, Corella D, de la Torre R, MartinezGonzalez MA, et al. Mediterranean diet and age-related cognitive decline: a randomized clinical trial. JAMA Intern Med. 2015;175(7):1094-103.

9. Park YM, Steck SE, Fung TT, Zhang J, Hazlett L, Han K, et al. Mediterranean diet, Dietary Approaches to Stop Hypertension (DASH) style diet, and metabolic health in U.S. adults. Clin Nutr. 2017;36(5):1301-9.

10. Reedy J, Lerman JL, Krebs-Smith SM, Kirkpatrick SI, Pannucci TE, Wilson MM, et al. Evaluation of the Healthy Eating Index-2015. J Acad Nutr Diet. 2018; 118(9):1622-33.

11. Krebs-Smith SM, Pannucci TE, Subar AF, Kirkpatrick SI, Lerman JL, Tooze JA, et al. Update of the Healthy Eating Index: HEl-2015. J Acad Nutr Diet. 2018; 118(9):1591-602.

12. Shivappa N, Hebert JR, Marcos A, Diaz L-E, Gomez S, Nova E, et al. Association between dietary inflammatory index and inflammatory markers in the HELENA study. Mol Nutr Food Res. 2017;61(6):https://doi.org/10.1002/ mnfr.201600707.

13. Namazi N, Larijani B, Azadbakht L. Association between the dietary inflammatory index and the incidence of cancer: a systematic review and meta-analysis of prospective studies. Public Health. 2018;164:148-56.

14. Phillips CM, Chen LW, Heude B, Bernard JY, Harvey NC, Duijts L, et al. Dietary Inflammatory Index and Non-Communicable Disease Risk: A Narrative Review. Nutrients. 2019;11(8):1873.

15. Shivappa N, Godos J, Hebert JR, Wirth MD, Piuri G, Speciani AF, Grosso G. Dietary inflammatory index and colorectal cancer risk-a meta-analysis. Nutrients. 2017;9(9):E1043.

16. Shivappa N, Godos J, Hebert JR, Wirth MD, Piuri G, Speciani AF, Grosso G. Dietary inflammatory index and cardiovascular risk and mortality-a metaanalysis. Nutrients. 2018;10(2):E200.

17. Garcia-Arellano A, Martinez-Gonzalez MA, Ramallal R, Salas-Salvado J, Hebert JR, Corella D, Shivappa N, Forga L, Schroder H, Munoz-Bravo C, Estruch R,
Fiol M, Lapetra J, Serra-Majem L, Ros E, Rekondo J, Toledo E, Razquin C, Ruiz-Canela M. Dietary inflammatory index and all-cause mortality in large cohorts: the SUN and PREDIMED studies. Clin Nutr. 2019;38(3):1221-31.

18. Kim D, Park Y. Association between the dietary inflammatory index and risk of frailty in older individuals with poor nutritional status. Nutrients. 2018;10(10):1363.

19. Zaslavsky O, Zelber-Sagi S, Hebert JR, Steck SE, Shivappa N, Tabung FK, Wirth MD, Bu Y, Shikany JM, Orchard T, Wallace RB, Snetselaar L, Tinker LF. Biomarker-calibrated nutrient intake and healthy diet index associations with mortality risks among older and frail women from the Women's Health Initiative. Am J Clin Nutr. 2017;105(6):1399-407.

20. Shivappa N, Stubbs B, Hebert JR, Cesari M, Schofield P, Soysal P, Maggi S, Veronese $\mathrm{N}$. The relationship between the dietary inflammatory index and incident frailty: a longitudinal cohort study. J Am Med Dir Assoc. 2018;19(1):77-82.

21. Lohman MC, Resciniti NV, Wirth MD, Shivappa N, Hebert JR. Obesity, dietary inflammation, and frailty among older adults: evidence from the National Health and Nutrition Examination Survey. J Nutr Gerontol Geriatrics. 2019; 38(1):18 -3218-32.

22. Resciniti NV, Lohman MC, Wirth MD, Shivappa N, Hebert JR. Dietary inflammatory index, pre-frailty and frailty among older US adults: evidence from the National Health and Nutrition Examination Survey, 2007-2014. J Nutr Health Aging. 2019;23(4):323-9.

23. Clegg A, Young J, lliffe S, Rikkert MO, Rockwood K. Frailty in elderly people. Lancet. 2013;381(9868):752-62.

24. Walters K, Frost R, Kharicha K, Avgerinou C, Gardner B, Ricciardi F, et al. Home-based health promotion for older people with mild frailty: the HomeHealth intervention development and feasibility RCT. Health Technol Assess. 2017;21 (73):1-128.

25. Blodgett JM, Theou O, Howlett SE, Rockwood K. A frailty index from common clinical and laboratory tests predicts increased risk of death across the life course. GeroScience. 2017;39(4):447-55.

26. Lorenzo-Lopez L, Maseda A, de Labra C, Regueiro-Folgueira L, RodriguezVillamil IL, Millan-Calenti JC. Nutritional determinants of frailty in older adults: a systematic review. BMC Geriatr. 2017;17(1):108.

27. Jayanama K, Theou O, Blodgett JM, Cahill L, Rockwood K. Frailty, nutrition-related parameters, and mortality across the adult age spectrum. BMC Med. 2018;16(1):188.

28. Theou O, Chapman I, Wijeyaratne L, Piantadosi C, Lange K, Naganathan V, et al. Can an intervention with testosterone and nutritional supplement improve the frailty level of under-nourished older people? J Frailty Aging. 2016;5(4):247-52.

29. Lo YL, Hsieh YT, Hsu LL, Chuang SY, Chang HY, Hsu CC, et al. Dietary pattern associated with frailty: results from nutrition and health survey in Taiwan. J Am Geriat Soc. 2017;65(9):2009-15.

30. Machón M, Mateo-Abad M, Vrotsou K, Zupiria X, Güell C, Rico L, et al. Dietary patterns and their relationship with frailty in functionally independent older adults. Nutrients. 2018;10(4):406.

31. National Center for Health Statistics (NCHS) of the Centers for Disease Control and Prevention (CDC). [cited 1 Oct 2018]. Available from: https//umw.cdcgov/nchs/nhanes/.

32. Zipf G, Chiappa M, Porter KS, Ostchega Y, Lewis BG, Dostal J. National health and nutrition examination survey: plan and operations, 1999-2010. Vital Health Stat 1. 2013;(56):1-37.

33. Jayanama K, Theou O, Blodgett JM, Cahill L, Rockwood K. Correction to: Frailty, nutrition-related parameters, and mortality across the adult age spectrum. BMC Med. 2018;16(1):235.

34. Shivappa N, Steck SE, Hurley TG, Hussey JR, Hebert JR. Designing and developing a literature-derived, population-based dietary inflammatory index. Public Health Nutr. 2014;17(8):1689-96.

35. Harmon BE, Wirth MD, Boushey CJ, Wilkens LR, Draluck E, Shivappa N, Steck SE, Hofseth L, Haiman CA, Le Marchand L, Hebert JR. The dietary inflammatory index is associated with colorectal cancer risk in the multiethnic cohort. J Nutr. 2017;147(3):430-8.

36. Hebert JR, Shivappa N, Wirth MD, Hussey JR, Hurley TG. Perspective: the Dietary Inflammatory Index (DI॥): lessons learned, improvements made and future directions. Adv Nutr. 2019;10(2):185-95.

37. Shivappa N, Wirth MD, Murphy EA, Hurley TG, Hébert JR. Association between the Dietary Inflammatory Index (DII) and urinary enterolignans and C-reactive protein from the National Health and Nutrition Examination Survey-2003-2008. Eur J Nutr. 2019;58(2):797-805. https://doi.org/10.1007/ s00394-018-1690-5.

38. U.S. Department of Health and Human Services and U.S. Department of Agriculture. 2015-2020 Dietary Guidelines for Americans. 8th Edition. December 2015. [lnternet]. [cited 1 Oct 2018]. Available from: https:/health. gov/dietaryguidelines/2015/guidelines/. 
39. Panagiotakos DB, Pitsavos C, Stefanadis C. Dietary patterns: a Mediterranean diet score and its relation to clinical and biological markers of cardiovascular disease risk. Nutr Metab Cardiovasc Dis. 2006;16(8):559-68.

40. Trichopoulou A, Costacou T, Bamia C, Trichopoulos D. Adherence to a Mediterranean diet and survival in a Greek population. N Engl J Med. 2003; 348(26):2599-608.

41. Z Zbeida M, Goldsmith R, Shimony T, Vardi H, Naggan L, Shahar DR. Mediterranean diet and functional indicators among older adults in non-Mediterranean and Mediterranean countries. J Nutr Health Aging. 2014;18(4):411-8.

42. Park Y-M, Zhang J, Steck SE, Fung TT, Hazlett L, Han K, et al. Obesity mediates the association between Mediterranean diet consumption and insulin resistance and inflammation in US adults. J Nutr. 2017;147(4):563-71.

43. Fung TT, McCullough ML, Newby PK, Manson JE, Meigs JB, Rifai N, et al. Diet-quality scores and plasma concentrations of markers of inflammation and endothelial dysfunction. Am J Clin Nutr. 2005;82(1):163-73.

44. Sacks FM, Svetkey LP, Vollmer WM, Appel LJ, Bray GA, Harsha D, et al. Effects on blood pressure of reduced dietary sodium and the Dietary Approaches to Stop Hypertension (DASH) diet. DASH-Sodium Collaborative Research Group. N Engl J Med. 2001;344(1):3-10.

45. Mellen PB, Gao SK, Vitolins MZ, Goff DC Jr. Deteriorating dietary habits among adults with hypertension: Dash dietary accordance, NHANES 19881994 and 1999-2004. Arch Intern Med. 2008;168(3):308-14.

46. Powell-Wiley TM, Miller PE, Agyemang P, Agurs-Collins T, Reedy J. Perceived and objective diet quality in US adults: a cross-sectional analysis of the National Health and Nutrition Examination Survey (NHANES). Public Health Nutr. 2014;17(12):2641-9.

47. National Center for Health Statistics. Office of Analysis and Epidemiology, Public-use Linked Mortality File, 2015. Hyattsville, Maryland. [cite 8 March 2019]. (Available from: https:/www.cdc.gov/nchs/data-linkage/mortality-publichtm).

48. Bharadwaj S, Ginoya S, Tandon P, Gohel TD, Guirguis J, Vallabh H, et al. Malnutrition: laboratory markers vs nutritional assessment. Gastroenterol Rep (Oxf). 2016;4(4):272-80.

49. Guasch-Ferré M, Salas-Salvadó J, Ros E, Estruch R, Corella D, Fitó M, Martínez-González MA, et al. The PREDIMED trial, Mediterranean diet and health outcomes: how strong is the evidence? Nutr Metab Cardiovasc Dis. 2017;27(7):624-32.

50. Petersson SD, Philippou E. Mediterranean diet, cognitive function, and dementia: a systematic review of the evidence. Adv Nutr. 2016;7:889-904.

51. Talegawkar SA, Bandinelli S, Bandeen-Roche K, Milaneschi Y, Tanaka T, Semba RD, Guralnik JM, Ferrucci L. A higher adherence to a Mediterraneanstyle diet is inversely associated with the development of frailty in community-dwelling elderly men and women. J Nutr. 2012;142:2161-6.

52. Fung TT, Chiuve SE, McCullough ML, Rexrode KM, Logroscino G, Hu FB. Adherence to a DASH-style diet and risk of coronary heart disease and stroke in women. Arch Intern Med. 2008;168(7):713-20.

53. George SM, Ballard-Barbash R, Manson JE, Reedy J, Shikany JM, Subar AF, et al. Comparing indices of diet quality with chronic disease mortality risk in postmenopausal women in the Women's Health Initiative Observational Study: evidence to inform national dietary guidance. Am J Epidemiol. 2014; 180(6):616-25.

54. Tabung FK, Steck SE, Zhang J, Ma Y, Liese AD, Agalliu I, et al. Construct validation of the dietary inflammatory index among postmenopausal women. Ann Epidemiol. 2015;25(6):398-405.

55. Stringhini S, Zaninotto P, Kumari M, Kivimäki M, Lassale C, Batty GD. Socioeconomic trajectories and cardiovascular disease mortality in older people: the English Longitudinal Study of Ageing. Int J Epidemiol. 2018;47(1):36-46.

56. Granic A, Sayer AA, Robinson SM. Dietary Patterns, Skeletal Muscle Health, and Sarcopenia in Older Adults. Nutrients. 2019;11(4):745.

57. Soltani S, Chitsazi MJ, Salehi-Abargouei A. The effect of dietary approaches to stop hypertension (DASH) on serum inflammatory markers: a systematic review and meta-analysis of randomized trials. Clin Nutr. 2018;37(2):542-50.

\section{Publisher's Note}

Springer Nature remains neutral with regard to jurisdictional claims in published maps and institutional affiliations.

\section{Ready to submit your research? Choose BMC and benefit from:}

- fast, convenient online submission

- thorough peer review by experienced researchers in your field

- rapid publication on acceptance

- support for research data, including large and complex data types

- gold Open Access which fosters wider collaboration and increased citations

- maximum visibility for your research: over $100 \mathrm{M}$ website views per year

At BMC, research is always in progress.

Learn more biomedcentral.com/submissions 